\title{
Jogos sérios e gamificação: um novo modelo para educação em saúde
}

\author{
Serious games and gamification: a new model for health education
}

Juegos serios y gamificación: un nuevo modelo de educación para la salud

\begin{abstract}
Thiago Alves Hungaro ${ }^{1 *}$, Ana Carolina Zanin Sacoman Kurihara ${ }^{1}$, Alexia Scavassa Pereira ${ }^{1}$, Kalebi Saraiva ${ }^{1}$.
\end{abstract}

\section{RESUMO}

Objetivo: Avaliar a utilização, aplicabilidade, acessibilidade e efetividade de recursos da gamificação para melhora da prevenção e promoção de saúde dentro da população geral, isto a fim de orientar novas pesquisas. Métodos: Foi utilizado o banco de dados Pubmed para revisão sistemática dos artigos, que se encaixavam nos critérios de inclusão e exclusão e análise de dados estatísticos fornecidos pela plataforma. Resultados: Após a aplicação dos critérios de inclusão e exclusão e leitura dos artigos, 38 foram selecionados. As categorias assunto principal, tipo de estudo, país/região como assunto, idioma do artigo, nome da revista e ano de publicação foram analisadas; sendo encontrados diferentes resultados em diferentes populações dentro da prevenção primária, secundária e terciária. Considerações finais: $\mathrm{Na}$ revisão foram observadas fragilidades como o número pequeno de amostragem, utilização de população homogênea e tempo curto de duração da intervenção. Observou-se também alguns resultados positivos, obtidos na análise objetiva do conhecimento dos grupos através de testes e pela devolutiva dos participantes.

Palavras-chave: Jogo, Educação, Gamificação.

\begin{abstract}
Objective: To evaluate the use, applicability, accessibility and effectiveness of gamification resources to improve prevention and health promotion within the general population, in order to guide further research. Methods: The Pubmed database was used for a systematic review of articles that met the inclusion and exclusion criteria and analysis of statistical data provided by the platform. Results: 39 articles were selected by reading the articles after applying the inclusion and exclusion criteria. Thus, it led us to analyze the main subject categories, type of study, country/region as subject, article language, journal name and year of publication. Showing different results in different populations within primary, secondary and tertiary prevention. Final considerations: In the review, weaknesses such as the small sample size, use of a homogeneous population and short duration of the intervention were observed. There were also some positive results, obtained in the objective analysis of the knowledge of the groups through tests and feedback from the participants.
\end{abstract}

Keywords: Game, Education, Gamification.

\section{RESUMEN}

Objetivo: Evaluar el uso, aplicabilidad, accesibilidad y efectividad de los recursos de gamificación para mejorar la prevención y promoción de la salud en la población general, con el fin de orientar futuras investigaciones. Métodos: Se utilizó la base de datos Pubmed para la revisión sistemática de artículos que cumplieron con los criterios de inclusión y exclusión y análisis de datos estadísticos proporcionados por la

${ }^{1}$ Centro Universitário Municipal de Franca (Uni-FACEF), Franca - SP. *E-mail: thungaro@hotmail.com

PUBLICADO EM: 9/2021 
plataforma. Resultados: Se seleccionaron 39 artículos mediante la lectura de los artículos luego de aplicar los criterios de inclusión y exclusión. Así, nos llevó a analizar las principales categorías temáticas, tipo de estudio, país / región como materia, idioma del artículo, nombre de la revista y año de publicación. Mostrando resultados diferentes en diferentes poblaciones dentro de la prevención primaria, secundaria y terciaria. Consideraciones finales: En la revisión se observaron debilidades como el pequeño tamaño de la muestra, el uso de una población homogénea y la corta duración de la intervención. También hubo algunos resultados positivos, obtenidos en el análisis objetivo de los conocimientos de los grupos a través de pruebas y retroalimentación de los participantes.

Palabras clave: Juego, Educación, Gamificación.

\section{INTRODUÇÃO}

O conceito de gamification surgiu recentemente, descrito como a aplicação de elementos de design de jogos em um contexto comum para motivar ou influenciar a participação e, também, se refere à criação de novos jogos sérios (ALLAN A, et al., 2015). Tornar a ciência mais acessível e interessante para o público através do processo de gamificação está cada vez mais popular nos últimos anos (COIL DA, et al., 2017).

Parte do processo de aprendizagem é tornar concreta sua evolução seja por notas, devolutivas entre outras. Dessa forma, para possibilitar correções ao decorrer da interação com o conhecimento, a gamificação está usando elementos de design de jogos, como: pontos, quadros de líderes, níveis, competições, recompensas, conquistas, mini-jogos, objetivos, pontos de experiência, regras, narrativa, gráficos, imaginação, identificação de papéis ou definição de desafios passo a passo, em busca de um objetivo (KLAASSEN R, et al., 2018).

Com o evoluir da tecnologia estamos entrando em contato com informações novas o tempo todo, porém, a retenção de conhecimento é prejudicada seja pela instantaneidade, pelo volume ou pelo método que estas são veiculadas. A aprendizagem baseada em jogos é um tipo de jogo e conteúdo de jogos que aprimora a aquisição de conhecimento, com o objetivo de alcançar um resultado definido de ensino e aprendizagem (HARUNA H, et al., 2018).

Um estudo realizado com pacientes diabéticos mostrou que o jogo pode fornecer informações detalhadas para os profissionais de saúde sobre as necessidades dos pacientes, fazendo com que 0 cuidado fosse centrado na pessoa e não no profissional, com melhor adesão aos cuidados, aumentando adesão a atividades físicas e melhores resultados laboratoriais, como a $\mathrm{HbA} 1 \mathrm{c}$ desse grupo quando comparado ao controle (STENOV V, et al., 2021).

Através de uma consulta na base de dados de artigos científicos, esse artigo procurou definir como métodos de ensino com linguagem atraente visualmente, dinâmicos cognitivamente, de fácil acesso e estimulantes, como os jogos, podem ser efetivos no ensino em saúde de populações gerais a fim de alcançar melhores resultados em comportamentos de prevenção e promoção de saúde.

\section{MÉTODOS}

A fim de se compilar dados sobre um determinado tema, pode-se utilizar os tipos de estudo como as revisões, dessa forma a revisão sistemática é uma boa forma de se sintetizar um tema através da pesquisa em uma fonte de dados. Pautada em uma pesquisa em uma data específica, a revisão sistemática procura abordar um tema específico de forma objetiva para que seja reprodutível futuramente, tornando-o um método robusto e quantitativo.

Este é um estudo qualitativo, retrospectivo e documental. Foi utilizado como ferramenta de pesquisa no dia 12/05/2021 o site da Biblioteca Virtual em saúde (BVSALUD) usando os termos "game", "education" e "gamification", totalizando 156 artigos. Foram selecionados artigos da base de dados Medical Literature Analysis and Retrieval System Online (MEDLINE) através do filtro da plataforma, assim totalizando 149 artigos. 
Neste período de maio foram aplicados os critérios de inclusão: artigos que falassem sobre prevenção e promoção em saúde, que contivessem resumo e estivessem no idioma inglês ou português. Também foram aplicados critérios de exclusão, retirando artigos que abordassem a formação no ensino superior, fossem de outras áreas que não a saúde e artigos sobre saúde não humana.

Após a aplicação do critério de inclusão do idioma foram encontrados 143 artigos. Após a inclusão dos demais critérios e leitura dos artigos foi observado que 105 artigos não se encaixavam nos critérios de inclusão e exclusão propostos para esta revisão sistemática, restando assim 38 artigos para análise.

\section{RESULTADOS}

Conforme exposto na Figura 1, após a aplicação dos critérios de inclusão e exclusão foram encontrados 38 artigos.

Figura 1 - Fluxograma de pesquisa



Fonte: Hungaro TA, et al., 2021.

As sínteses dos artigos encontrados na revisão, contendo um resumo dos objetivos, resultados e conclusão dos artigos analisados, encontram-se no Quadro 1. 
Quadro 1 - Síntese dos 38 artigos utilizados na revisão.

\begin{tabular}{|c|c|c|c|}
\hline $\mathbf{N}$ & Autores (Ano) & Tipo de estudo & Objetivo \\
\hline 1 & $\begin{array}{l}\text { STENOV V, et al. } \\
\qquad(2021)\end{array}$ & Pesquisa qualitativa & $\begin{array}{l}\text { Explorar o resultado do uso de um jogo } \\
\text { analógico no apoio ao paciente com diabetes } \\
\text { mellitus. }\end{array}$ \\
\hline 2 & $\begin{array}{l}\text { DARVILLE G, et } \\
\text { al. }(2021)\end{array}$ & Pesquisa qualitativa & $\begin{array}{l}\text { Relacionar o uso de gamificação com a } \\
\text { conscientização da vacina contra o HPV. }\end{array}$ \\
\hline 3 & $\begin{array}{l}\text { GASPAR JDS, et } \\
\text { al. (2020) }\end{array}$ & $\begin{array}{c}\text { Estudo } \\
\text { observacional }\end{array}$ & $\begin{array}{l}\text { Desenvolver um jogo sério que forneça } \\
\text { informações com evidências científicas sobre } \\
\text { a prevenção da COVID-19 e cuidados } \\
\text { pessoais durante a pandemia, a partir da } \\
\text { testagem de conhecimento dos jogadores. }\end{array}$ \\
\hline 4 & $\begin{array}{l}\text { MARTOS- } \\
\text { CABRERA MB } \\
(2020)\end{array}$ & Estudo coorte & $\begin{array}{l}\text { Avaliar o efeito dos jogos sobre o nível de } \\
\text { hemoglobina glicada de paciente diabéticos }\end{array}$ \\
\hline 5 & $\begin{array}{l}\text { HUYNH E, et al. } \\
(2020)\end{array}$ & Estudo coorte & $\begin{array}{l}\text { Mostrar como jogos podem ajudar na } \\
\text { alfabetização de crianças pequenas. }\end{array}$ \\
\hline 6 & $\begin{array}{l}\text { POURBASSI A, et } \\
\text { al. (2020) }\end{array}$ & Caso controle & $\begin{array}{l}\text { Avaliar um modelo de atividade física, que é } \\
\text { promovido por meio de uma brincadeira entre } \\
\text { alunos do ensino médio. }\end{array}$ \\
\hline 7 & $\begin{array}{l}\text { FIJACKO N, et al. } \\
\qquad(2020)\end{array}$ & $\begin{array}{c}\text { Estudo } \\
\text { observacional }\end{array}$ & $\begin{array}{l}\text { Identificar aplicativos de } \\
\text { smartphone/gamificação voltados para motivar } \\
\text { crianças a aprender, realizar e manter uma } \\
\text { saúde bucal ideal. }\end{array}$ \\
\hline 8 & $\begin{array}{l}\text { ZAKARIA NS, et } \\
\quad \text { al. }(2020)\end{array}$ & Revisão sistemática & $\begin{array}{l}\text { Apresentar o Ethoshunt como um aplicativo } \\
\text { móvel baseado em gamificação que pode ser } \\
\text { usado no ensino e aprendizagem de ética. }\end{array}$ \\
\hline 9 & $\begin{array}{l}\text { QUINTAS-HIJÓS } \\
\text { A, et al. (2020) }\end{array}$ & Estudo prognóstico & $\begin{array}{l}\text { Uso de jogos (gamification + exergames) } \\
\text { como didática em aulas de Educação física } \\
\text { (EF) para aumentar a performance acadêmica } \\
\text { e motivar o hábito em atividade física. }\end{array}$ \\
\hline
\end{tabular}

\section{Principais achados}

As respostas ao questionário mostraram que $92 \%$ das pescom diálogo com os pares na educacão. foco na pessoa e diálogo com os pares na educação.

Modelos teóricos de conscientização são eficientes, assim como modelos em gamificação e para isso medidas dentro e fora do jogo foram discutidas como parâmetros de impacto.

A taxa de erro no tópico "máscara" mostrou tendência negativa $e$ os outros tópicos não demonstraram endências significativas. $O$ acesso ao jogo aumentou com o passar dos dias e aumento da divulgação.

Utilizando jogos eletrônicos e físicos houve melhora dos níveis de hemoglobina glicada dos pacientes em comparação ao grupo que não utilizou de nenhum tipo de jogo. Apesar de os alunos precisarem de o dobro de tempo para completar a tarefa, isso não prejudicou a avaliação. abilide habilidades de leitura de gráficos, mas diferenças significativas no envolvimento e na diversão geral do jogo. Os pacientes que utilizaram o jogo conseguiram se lembrar dos hábitos saudáveis e de atividade física, mais do que os pares do grupo controle.

Os recursos de gamificação mais utilizados foram contagem de tempo, personagens virtuais e fantasias, com boa qualidade. Influência para mudança de comportamento foram detectadas em todos os aplicativos.

fluxo de aprendizagem pelo Ethoshunt foi discutido No geral, os resultados foram positivos, e a usabilidade de aplicativos móveis teve a ração mais forte com emoçães positivas seguido por competência ética e experiencia do positivas, seguido por competencia ética experiência do feedback foi através de avaliações positivas (28,6\%), ceu caráter molvador (28,1\%) e in formalivo (13\%), ou sua comentários negativos referem-se a sentimento de indiferenç $(6,3 \%)$ estresse $(4,7 \%)$, frustração $(2,6 \%)$ ou besteira $(2,1 \%)$. As opiniões mais frequentes introducão \% . As opinio istroduçao do exergame nas aulas de EF foram inespecífica $(21,9 \%)$, variedade de músicas $(18,2 \%)$ inespecifica $(21,9 \%)$, variedade de músicas $(18,2 \%)$

\section{Conclusão}

O jogo proporcionou a centralização do cuidado no paciente, jogos semelhantes podem ser utilizados para aprimorar o cuidado do paciente.

As intervenções em conscientização devem ter enfoque em utilizar mais vezes jogos sérios, pois a incorporação de teoria e métodos padronizados podem encorajar homens a se vacinarem contra o HPV.

Atingiu mais o público jovem, prioritário na estratégia de orientação para o distanciamento social. A melhoria do desempenho dos participantes pode refletir um aumento nas informações.

Foi constatado efeito positivo nos pacientes, mas o impacto é baixo para melhorar os níveis de hemoglobina glicada.

Todas as crianças no grupo de idade mais jovem melhoraram ou exibiram nenhuma mudança no desempenho, ao contránio de outras faixas elárias. Isso, combinado com comentários de que o jogo seria mais adequado para novos alunos.

A gamificação pode melhorar significativamente a eficácia da educação para atividade física.

A maioria dos aplicativos de higiene bucal analisados, inclui recursos de gamificação e técnicas de mudança de comportamento, para realizar e manter a higiene bucal em crianças. Com conteúdo odontológico baseado em evidências.

O aplicativo móvel baseado em gamificação defendido neste estudo pode fornecer ideias para educadores de ética que desejam desenvolver um ambiente de aprendizagem mediado por tecnologia.

Não obteve resultado conclusivo sobre o impacto em atividade física. Aqueles que já praticavam atividade física preferiram outra ativida pran nã envolvessem tecnologia sedentários relataram que não fariam em casa. Aqueles familiarizados com tecnologia receberam uma dinâmica melhor. 


\begin{tabular}{|c|c|c|c|c|c|}
\hline $\mathbf{N}$ & Autores (Ano) & Tipo de estudo & Objetivo & Principais achados & Conclusão \\
\hline 10 & $\begin{array}{l}\text { VILLASANA MV, } \\
\text { et al. }(2020)\end{array}$ & Revisão sistemática & $\begin{array}{l}\text { Revisão entre } 2009 \text { e } 2019 \text { para avaliar qual a } \\
\text { metodologia mais usada em aplicativos de } \\
\text { aparelhos móveis para promoção de hábitos } \\
\text { saudáveis em adolescentes, como eles o } \\
\text { fazem e se são avaliados positivamente por } \\
\text { estes. }\end{array}$ & $\begin{array}{l}\text { Houve concordância na melhora em hábitos alimentares } \\
\text { em alguns deles, assim como na atividade física. Houve } \\
\text { uma avaliação positiva pelos adolescentes em } 66,67 \% \\
\text { dos artigos inclusos. }\end{array}$ & $\begin{array}{l}\text { O uso de gamificação e questionários cativa os } \\
\text { adolescentes a usarem aplicativos móveis para a } \\
\text { promoção de hábitos alimentares saudáveis e } \\
\text { físicos }\end{array}$ \\
\hline 11 & $\begin{array}{l}\text { CEJUDO J, et al. } \\
(2020)\end{array}$ & Pesquisa qualitativa & $\begin{array}{l}\text { Procurou avaliar o uso de jogos na melhora da } \\
\text { inteligência emocional, competências sociais e } \\
\text { bem-estar social em adolescentes. }\end{array}$ & $\begin{array}{l}\text { Os resultados das análises pós-teste de covariância (co- } \\
\text { variando as pontuaçốes pré-teste) mostraram que os } \\
\text { participantes do grupo experimental aumentaram } \\
\text { significativamente as pontuações em Qualidade de Vida } \\
\text { Relacionada à Saúde, Afeto Positivo e Saúde Mental. }\end{array}$ & $\begin{array}{l}\text { O uso do jogo se provou uma estratégia educacional } \\
\text { efetiva na melhora de habilidades pessoais e } \\
\text { sociais. Se mostrou benéfico em prevenir doenças } \\
\text { mentais, que limitam a habilidade dos adolescentes } \\
\text { em desenvolver relacionamentos positivos e assim } \\
\text { sua performance acadêmica e social. }\end{array}$ \\
\hline 12 & $\begin{array}{l}\text { TALLEY MH, et al. } \\
\text { (2019) }\end{array}$ & Estudo coorte & $\begin{array}{l}\text { Procurou saber o que pacientes adultos com } \\
\text { diabetes acham do uso de educação } \\
\text { gamificada no contexto da automanutenção da } \\
\text { diabetes. }\end{array}$ & $\begin{array}{l}\text { Pacientes relataram boa adesão com desejo de jogar } \\
\text { novamente com os familiares com boa distribuição de } \\
\text { dificuldade das questões. }\end{array}$ & $\begin{array}{l}\text { Obteve um ótimo retorno com os pacientes, que } \\
\text { relataram se sentir mais empoderados com a própria } \\
\text { doença, para falar com seus provedores de saude. } \\
\text { Levou-os a mudar a dinâmica do atendimento, } \\
\text { resultando em uma "consulta inversa", semelhante a } \\
\text { aula inversa, o foco do aprendizado é o paciente. }\end{array}$ \\
\hline 13 & $\begin{array}{l}\text { MONTANER J, et } \\
\text { al. (2019) }\end{array}$ & Estudo coorte & $\begin{array}{l}\text { Através de uma dinâmica envolvendo } \\
\text { gamification com tablets e mecatrônica, } \\
\text { procurou avaliar a influência positiva de } \\
\text { conversação, coordenação motora e estado } \\
\text { psicossocial de crianças entre } 4 \text { a } 12 \text { anos } \\
\text { internadas em hospital. }\end{array}$ & $\begin{array}{l}\text { Mostrou diferenças estatisticamente significativas para } \\
\text { afeto, atividade e interação social. Isso mostra um impacto } \\
\text { de curto prazo no estado psicossocial dos participantes. }\end{array}$ & $\begin{array}{l}\text { Verificou-se uma melhora no quesito de } \\
\text { comunicação e coordenação pela interação com o } \\
\text { professor que permanecia na dinâmica. A melhora } \\
\text { psicossocial foi exemplificada pela própria atividade } \\
\text { que, mesmo sem a interação do professor, mostrou } \\
\text { melhora da feição após alguns minutos de jogo e } \\
\text { manteve o paciente entretido, os fazendo esquecer } \\
\text { momentaneamente dos seus sintomas. }\end{array}$ \\
\hline 14 & $\begin{array}{l}\text { HOLZMANN SL, } \\
\text { et al. (2019) }\end{array}$ & $\begin{array}{l}\text { Estudo de } \\
\text { prevalência }\end{array}$ & $\begin{array}{l}\text { Comparou a efetividade do uso do jogo sério } \\
\text { no conhecimento nutricional em estudantes do } \\
\text { fundamental em comparação a uma aula } \\
\text { comum a curto prazo. }\end{array}$ & $\begin{array}{l}\text { Obteve-se melhora do conhecimento sobre nutrição no } \\
\text { grupo com jogo, porém maior melhora no grupo com } \\
\text { aulas. }\end{array}$ & $\begin{array}{l}\text { O artigo mostrou limitações como amostra pequena, } \\
\text { com maioria masculina e avaliadores possivelmente } \\
\text { não imparciais nas aulas ministradas no grupo sem } \\
\text { jogo. Não foi possível estabelecer mudança de } \\
\text { comportamento, mas não ficou determinada sua } \\
\text { negativa em uma pesquisa longitudinal. }\end{array}$ \\
\hline 15 & $\begin{array}{l}\text { OTERO-AGRA M, } \\
\text { et al. (2019) }\end{array}$ & Caso controle & $\begin{array}{l}\text { Uso de gamificação para avaliar a efetividade } \\
\text { da qualidade da Ressuscitação } \\
\text { Cardiopulmonar (RCP) em estudantes do } \\
\text { fundamental. }\end{array}$ & $\begin{array}{l}\text { O método de treinamento baseado na gamificação } \\
\text { resultou em melhores scores na qualidade da RCP } \\
\text { ( } 89.56 \% ; 95 \% \mathrm{Cl} 86.71 \text { a } 92.42) \text { do que o método com } \\
\text { evolução }(81.96 \% ; 95 \% \mathrm{Cl} 78.04 \% \text { a } 85.88 \%) \text {. }\end{array}$ & $\begin{array}{l}\text { Obteve-se melhor qualidade de CPR no grupo de } \\
\text { treinamento com gamificação do que no grupo de } \\
\text { treinamento com evolução, mostrando que a } \\
\text { gamificação é um recurso pedagógico de ensino } \\
\text { com bons resultados. }\end{array}$ \\
\hline 16 & $\begin{array}{l}\text { BLOK AC, et al. } \\
(2019)\end{array}$ & Caso controle & $\begin{array}{l}\text { Uso de aplicativo móvel por } 3 \text { semanas para } \\
\text { motivar } 41 \text { tabagistas, em etapa de } \\
\text { contemplação, e preparar eles com confiança } \\
\text { e habilidades necessárias para ser bem- } \\
\text { sucedido quando evoluir para fase de ação. }\end{array}$ & $\begin{array}{l}\text { 84,7\% dos participantes responderam continuamente aos } \\
\text { quizzes enviados por sms, e } 83 \% \text { relataram que as } \\
\text { mensagens motivacionais foram de grande ajuda. Alguns } \\
\text { participantes definiram uma data para cessar o tabagismo, } \\
\text { mesmo que por um pequeno período, no desafio de } 2 \\
\text { semanas. }\end{array}$ & $\begin{array}{l}\text { Mostrou que com a gamificação há um estímulo } \\
\text { constante para incorporar comportamentos } \\
\text { baseados em evidência, no processo de cessação } \\
\text { de tabagismo, principalmente em pacientes com } \\
\text { alguma vulnerabilidade social. }\end{array}$ \\
\hline 17 & $\begin{array}{l}\text { AZEVEDO J, et al. } \\
(2019)\end{array}$ & $\begin{array}{l}\text { Estudo } \\
\text { observacional }\end{array}$ & $\begin{array}{l}\text { Usou um jogo via navegador para melhorar o } \\
\text { entendimento nutricional de famílias de } \\
\text { crianças entre } 3 \text { a } 5 \text { anos. }\end{array}$ & $\begin{array}{l}\text { Usou uma amostra de } 37 \text { salas de jardim de infância, } \\
\text { totalizando } 877 \text { famílias, com } 32 \text { salas de jardim de } \\
\text { infância sendo do grupo de intervenção e } 5 \text { do grupo de } \\
\text { controle. Apenas } 106 \text { famílias do grupo de intervenção e } \\
83 \text { do grupo de controle completaram o teste. }\end{array}$ & $\begin{array}{l}\text { Mostrou resultados positivos com o grupo com } \\
\text { intervenção sendo melhor avaliado ao final da } \\
\text { pontuação. Proporcionou boa difusão das } \\
\text { informações através da interação entre os } \\
\text { participantes. }\end{array}$ \\
\hline 18 & $\begin{array}{l}\text { HARUNA H, et al. } \\
(2019)\end{array}$ & Estudo transversal & $\begin{array}{l}\text { Avaliar a eficácia de jogos de ensino em } \\
\text { instruir adolescentes sobre a saúde sexual, e } \\
\text { se levam à mudança no comportamento ou } \\
\text { não, a partir da estrutura MAKE (motivação, } \\
\text { atitude, conhecimento e engajamento). }\end{array}$ & $\begin{array}{l}\text { A estrutura MAKE é abrangente para avaliar métodos de } \\
\text { ensino. }\end{array}$ & É um instrumento satisfatório. \\
\hline
\end{tabular}




\begin{tabular}{|c|c|c|c|c|c|}
\hline $\mathbf{N}$ & Autores (Ano) & Tipo de estudo & Objetivo & Principais achados & Conclusão \\
\hline 19 & $\begin{array}{l}\text { BELOGIANNI Ke } \\
\text { BALDWIN C } \\
(2019)\end{array}$ & $\begin{array}{l}\text { Estudo de } \\
\text { prevalência }\end{array}$ & $\begin{array}{l}\text { Avaliar o impacto de jogos educativos no } \\
\text { contexto nutricional, de hábitos alimentares e } \\
\text { de atividade física em universitários do Reino } \\
\text { Unido. }\end{array}$ & $\begin{array}{l}\text { As mudanças a partir do conhecimento foram avaliadas } \\
\text { por correlações. }\end{array}$ & $\begin{array}{l}\text { Uma abordagem interessante para melhorar o } \\
\text { conhecimento nutricional desse público. }\end{array}$ \\
\hline 20 & $\begin{array}{l}\mathrm{RYU} \mathrm{JH}, \text { et al. } \\
(2018)\end{array}$ & $\begin{array}{l}\text { Estudo de } \\
\text { prevalência }\end{array}$ & $\begin{array}{l}\text { Avaliar se a gamificacãa do processo pré- } \\
\text { operatório por meio de jogos com realidade } \\
\text { virtual pode reduzir a ansiedade de crianças } \\
\text { pré cirurgias. }\end{array}$ & $\begin{array}{l}\text { A partir das crianças separadas aleatoriamente em } \\
\text { controle e gamificação, as informações pré-anestésicas } \\
\text { foram passadas por método convencional e gamificação, } \\
\text { respectivamente, e a ansiedade foi medida por escalas } \\
\text { validadas. }\end{array}$ & $\begin{array}{l}\text { O uso de realidade virtual pode reduzir a ansiedade } \\
\text { das crianças nesse momento e melhorar a adesão } \\
\text { durante a indução anestésica. }\end{array}$ \\
\hline 21 & $\begin{array}{l}\text { HARUNA H, et al. } \\
(2018)\end{array}$ & $\begin{array}{l}\text { Ensaio clínico } \\
\text { controlado }\end{array}$ & $\begin{array}{l}\text { Investigar até qual ponto os jogos educativos } \\
\text { podem melhorar a saúde sexual de } \\
\text { adolescentes. }\end{array}$ & $\begin{array}{l}\text { A partir de uma avaliação pela estrutura MAKE } \\
\text { (motivação, atitude, conhecimento e engajamento), bons } \\
\text { resultados foram encontrados para o uso de jogos } \\
\text { educativos. }\end{array}$ & $\begin{array}{l}\text { Os jogos podem contribuir para a melhoria do } \\
\text { conhecimento e comportamento relacionados à } \\
\text { saúde sexual, em uma região que vinha sofrendo } \\
\text { com surtos de AIDS/HIV. }\end{array}$ \\
\hline 22 & $\begin{array}{l}\text { ALMONDES KM e } \\
\text { LEONARDO MEM } \\
\text { (2018) }\end{array}$ & Estudo coorte & $\begin{array}{l}\text { Avaliar os efeitos de jogos sérios nas } \\
\text { recomendações de higiene do sono. }\end{array}$ & $\begin{array}{l}\text { A partir do uso do jogo "quarto perfeito" com instruções } \\
\text { para crianças sobre higiene do sono, utilizado em } 6 \\
\text { sessões de } 50 \text { minutos. Questionários e escalas foram } \\
\text { respondidos pelos responsáveis, para avaliar o impacto do } \\
\text { jogo. }\end{array}$ & $\begin{array}{l}\text { O jogo pode ser aplicado com a finalidade de } \\
\text { melhorar o sono e os impactos da qualidade do } \\
\text { sono em crianças, mas os resultados também } \\
\text { dependem dos hábitos familiares. }\end{array}$ \\
\hline 23 & $\begin{array}{l}\text { KLAASSEN R, et } \\
\text { al. (2018) }\end{array}$ & $\begin{array}{l}\text { Estudo } \\
\text { observacional }\end{array}$ & $\begin{array}{l}\text { Descrever uma plataforma que seja adequada } \\
\text { para o autocuidado de pacientes pediátricos } \\
\text { diabéticos. }\end{array}$ & $\begin{array}{l}\text { O uso de tecnologia combinada com conhecimento e } \\
\text { saúde tem bons resultados. }\end{array}$ & $\begin{array}{l}\text { Jogos sérios, quando planejados para a população } \\
\text { pediátrica, trazem mais eficiência para o } \\
\text { aprendizado e mudança de hábitos e cuidados do } \\
\text { que quando utilizados jogos não desenhados para } \\
\text { populações específicas. }\end{array}$ \\
\hline 24 & $\begin{array}{l}\text { STAPINSKI LA, et } \\
\text { al. (2018) }\end{array}$ & $\begin{array}{l}\text { Ensaio clínico } \\
\text { controlado }\end{array}$ & $\begin{array}{l}\text { Avaliar o uso do jogo "Pure Rush" para } \\
\text { abordar educação sobre drogas na Austrália. }\end{array}$ & $\begin{array}{l}\text { Resultados: os alunos gostaram de jogar "Pure Rush" e } \\
\text { acharam adequado para a idade deles, com informações } \\
\text { úteis. Foi evidenciado um aumento significativo do } \\
\text { conhecimento, por meio de pré e pós teste. }\end{array}$ & $\begin{array}{l}\text { É um método válido de ser implementado nas } \\
\text { escolas para melhorar o conhecimento sobre } \\
\text { drogas. }\end{array}$ \\
\hline 25 & $\begin{array}{l}\text { HIGHTOW- } \\
\text { WEIDMAN LB, et } \\
\text { al. (2017) }\end{array}$ & Revisão sistemática & $\begin{array}{l}\text { Fornecer um contexto histórico para o uso de } \\
\text { gamificação e videogame usados em } \\
\text { intervenções de HIV. }\end{array}$ & $\begin{array}{l}\text { Poucos recursos digitais são desenvolvidos para a } \\
\text { prevenção do HIV, mas um portfólio maior de intervenções } \\
\text { digitais está a caminho. }\end{array}$ & $\begin{array}{l}\text { Os jogos são estratégias promissoras para } \\
\text { prevenção e cuidado do HIV, principalmente para os } \\
\text { jovens, e, ainda há espaço para crescimento dessa } \\
\text { metodologia. }\end{array}$ \\
\hline 26 & $\begin{array}{l}\text { RYAN J, et al. } \\
(2017)\end{array}$ & $\begin{array}{l}\text { Estudo } \\
\text { observacional }\end{array}$ & $\begin{array}{l}\text { Examinou o envolvimento, conformidade e } \\
\text { retenção de informações de usuários do } \\
\text { "Active Team", uma intervenção para atividade } \\
\text { física, plataforma oferecida por meio de um } \\
\text { aplicativo online no facebook. }\end{array}$ & $\begin{array}{l}\text { As análises revelaram uma associação significativa entre } \\
\text { o envolvimento, o jogo e a educação dos participantes do } \\
\text { ensino médio. Mas há necessidade de adaptação para } \\
\text { cada público alvo. Além de que o uso de notificações, } \\
\text { como lembretes aumentou a adesão dos usuários. }\end{array}$ & $\begin{array}{l}\text { É uma boa plataforma para melhorar a adesão dos } \\
\text { usuários à prática de atividades físicas, desde que } \\
\text { seja adaptado para cada público alvo, os resultados } \\
\text { podem ser ainda melhores. }\end{array}$ \\
\hline 27 & $\begin{array}{l}\text { INGADOTTIR B, } \\
\text { et al. (2017) }\end{array}$ & Estudo coorte & $\begin{array}{l}\text { Descrever um desenvolvimento de um jogo } \\
\text { baseado em computador para pacientes } \\
\text { cirúrgicos para aprender o manejo de dor pós- } \\
\text { operatório, avaliando a usabilidade, } \\
\text { experiência e eficácia do jogo. }\end{array}$ & $\begin{array}{l}\text { Houve facilidade de uso, levando a satisfação dos } \\
\text { usuários com o novo método de aprendizagem, mesmo } \\
\text { com desafios tecnológicos. O conhecimento de medicação } \\
\text { para dor melhorou depois do jogo. }\end{array}$ & $\begin{array}{l}\text { Um jogo de computador pode ser um método } \\
\text { eficiente para aprender sobre o controle da dor. Mas } \\
\text { para avaliar a usabilidade e eficácia no contexto de } \\
\text { preparação para a cirurgia, uma amostra maior é } \\
\text { necessária. }\end{array}$ \\
\hline 28 & $\begin{array}{l}\text { COIL DA, et al. } \\
(2017)\end{array}$ & Estudo transversal & $\begin{array}{l}\text { Discutir o desenvolvimento de um jogo de } \\
\text { tabuleiro chamado "Gut Check: The } \\
\text { Microbioma Game" }\end{array}$ & $\begin{array}{l}\text { Os jogos de pedagogia científica têm potencial de } \\
\text { envolver e estimular os alunos, melhorando a retenção do } \\
\text { conhecimento, mas para isso devem ser divertidos e } \\
\text { desafiadores. }\end{array}$ & $\begin{array}{l}\text { Para que os jogos sejam mais eficientes, ou seja, } \\
\text { divertidos, adequados para o público alvo e } \\
\text { desafiadores, é necessário grande investimento de } \\
\text { tempo e dinheiro em produçăo e manufatura. }\end{array}$ \\
\hline 29 & $\begin{array}{l}\text { BRÜLL P, et al. } \\
\qquad(2016)\end{array}$ & Estudo transversal & $\begin{array}{l}\text { Educação sexual desenvolvida para } \\
\text { adolescentes e adultos jovens a fim promover } \\
\text { sexo mais seguro e diminuir o índice de IST } \\
\text { em pessoas jovens. }\end{array}$ & $\begin{array}{l}\text { Independente do histórico cultural ou do gênero houveram } \\
\text { demandas semelhantes para a abordagem de educação } \\
\text { sexual gamificada. }\end{array}$ & $\begin{array}{l}\text { Jogos educacionais oferecem anonimato e a } \\
\text { oportunidade de pessoas de diferentes históricos } \\
\text { culturais participarem de uma educação sexual em } \\
\text { um ambiente seguro e não ameaçador. }\end{array}$ \\
\hline 30 & $\begin{array}{l}\text { BURGESS JD, et } \\
\text { al. (2016) }\end{array}$ & $\begin{array}{l}\text { Estudo } \\
\text { observacional }\end{array}$ & $\begin{array}{l}\text { Usar um aplicativo de smartphone para } \\
\text { aumentar o conhecimento e a atenção quanto } \\
\text { aos riscos de escaldamento com bebidas } \\
\text { quentes e primeiros socorros a queimaduras } \\
\text { em mães de crianças pequenas. }\end{array}$ & $\begin{array}{l}499 \text { participantes foram recrutados até agora e o período } \\
\text { de teste está ativo. }\end{array}$ & Ainda não houve. \\
\hline
\end{tabular}




\begin{tabular}{|c|c|c|c|c|c|}
\hline $\mathbf{N}$ & Autores (Ano) & Tipo de estudo & Objetivo & Principais achados & Conclusão \\
\hline 31 & $\begin{array}{l}\text { RIORDAN BC, et } \\
\text { al. (2016) }\end{array}$ & Estudo coorte & $\begin{array}{l}\text { Avaliar o uso de minecraft para criar } \\
\text { comunidades e capacidade cognitiva. }\end{array}$ & $\begin{array}{l}\text { O autor abordou brevemente alguns usos do Minecraft } \\
\text { relacionado à educação, mas não aprofundou a discussão } \\
\text { em nenhum deles. }\end{array}$ & $\begin{array}{l}\text { Demonstrou um potencial importante no uso do jogo } \\
\text { Minecraft edição educativa para promover } \\
\text { socialização, bem estar e resiliência em diferentes } \\
\text { grupos. }\end{array}$ \\
\hline 32 & $\begin{array}{l}\text { FLEMING TM, et } \\
\text { al. (2016) }\end{array}$ & Revisão sistemática & $\begin{array}{l}\text { Revisão da situação atual do uso de jogos } \\
\text { para tratamento em saúde mental e direções } \\
\text { promissoras para novas pesquisas }\end{array}$ & $\begin{array}{l}\text { Atualmente são usados exergames, realidade virtual, } \\
\text { jogos baseados em terapia cognitiva- comportamental, } \\
\text { jogos de entretenimento, biofeedback e jogos de } \\
\text { treinamento cognitivo. }\end{array}$ & $\begin{array}{l}\text { Precisa-se de um maior desenvolvimento de } \\
\text { intervenções de saúde mental eficazes e atraentes } \\
\text { que alcancem um grande número de pessoas, } \\
\text { assim devem incluir testes independentes e } \\
\text { comparações diretas de opções baseadas em jogos } \\
\text { e não baseadas em jogos, para grupos de usuários } \\
\text { variados. }\end{array}$ \\
\hline 33 & $\begin{array}{l}\text { THENG YL, et al. } \\
\qquad(2015)\end{array}$ & Estudo de incidência & $\begin{array}{l}\text { Revisar a literatura que enfoca no uso de } \\
\text { videogames, métodos de gamificação e } \\
\text { ambientes virtuais, para o autogerenciamento } \\
\text { diário de pessoas com diabetes. }\end{array}$ & $\begin{array}{l}\text { Dos } 307 \text { artigos, apenas } 10 \text { se encaixaram nos critérios de } \\
\text { inclusão e } 4 \text { deles usaram jogos eletrônicos, } 3 \text { usaram } \\
\text { realidade virtual e } 3 \text { usaram princípios da gamificação. }\end{array}$ & $\begin{array}{l}\text { Obteve melhor controle de níveis glicêmicos após o } \\
\text { uso de um sistema com mecânica de recompensas. } \\
\text { O paciente que internaliza esses comportamentos } \\
\text { alcançou uma melhor adesão medicamentosa e } \\
\text { relação com profissionais de saúde. }\end{array}$ \\
\hline 34 & $\begin{array}{l}\text { DESMET A, et al. } \\
(2015)\end{array}$ & Estudo de incidência & $\begin{array}{l}\text { Meta análise para investigar a eficácia do uso } \\
\text { de jogos sérios para a promoção de saúde } \\
\text { sexual. }\end{array}$ & $\begin{array}{l}\text { Foram incluídos } 7 \text { estudos, estes mostraram efeitos } \\
\text { positivos baseados principalmente no uso de } \\
\text { componentes como recompensas e devolutiva. }\end{array}$ & $\begin{array}{l}\text { A próxima geração de jogos pode ser aprimorada } \\
\text { com base em mudança comportamental e em } \\
\text { literatura de jogos educacionais. Necessita-se de } \\
\text { estudos que avaliem os efeitos a mais longo prazo, } \\
\text { medidas de comportamento e sua eficácia. }\end{array}$ \\
\hline 35 & $\begin{array}{l}\text { ALLAN A, et al. } \\
(2015)\end{array}$ & Revisão sistemática & $\begin{array}{l}\text { Examinar os efeitos de uma intervenção } \\
\text { baseada na Web que incluiu recursos de } \\
\text { suporte social online e gamificação na } \\
\text { atividade física, utilização de cuidados de } \\
\text { saúde, uso excessivo de medicamentos, } \\
\text { capacitação e conhecimento de Artrite } \\
\text { Reumatóide (AR) de pacientes com AR. }\end{array}$ & $\begin{array}{l}\text { Houve melhora na prática de atividade física, diminuição } \\
\text { do uso de serviços de saúde e melhor autonomia da } \\
\text { doença. }\end{array}$ & $\begin{array}{l}\text { Potencial efeito positivo da gamificação e do suporte } \\
\text { social online na saúde e nos resultados } \\
\text { comportamentais. }\end{array}$ \\
\hline 36 & $\begin{array}{l}\text { JONES BA, et al. } \\
\qquad(2014)\end{array}$ & Estudo coorte & $\begin{array}{l}\text { Usou uma abordagem de gamificação } \\
\text { baseada em comportamento para desenvolver } \\
\text { um aumento do consumo de frutas e vegetais } \\
\text { (FV), também minimizando os custos de } \\
\text { material e mão de obra para a escola. }\end{array}$ & $\begin{array}{l}\text { O consumo de FV aumentou acima dos níveis basais em } \\
66 \% \text { e } 44 \% \text {, respectivamente, quando medido em xícaras, } \\
\text { e } 84 \% \text { e } 28 \% \text {, respectivamente, quando medido em } \\
\text { gramas. É importante ressaltar que, como o consumo do } \\
\text { alimento alvo (frutas ou vegetais) aumentou } \\
\text { significativamente acima dos níveis basais, mas o } \\
\text { consumo do alimento não direcionado não, os aumentos } \\
\text { gerais no consumo de FV podem ser atribuídos à eficácia } \\
\text { da intervenção. }\end{array}$ & $\begin{array}{l}\text { Demonstrou a viabilidade e eficácia iniciais de uma } \\
\text { intervenção baseada em gamificação para aumentar } \\
\text { o consumo de FV em toda a escola. }\end{array}$ \\
\hline 37 & $\begin{array}{l}\text { GABARRON E, et } \\
\text { al. (2013) }\end{array}$ & $\begin{array}{c}\text { Estudo } \\
\text { observacional }\end{array}$ & $\begin{array}{l}\text { Uso de um jogo via web para ensino em } \\
\text { saúde sexual a fim de aumentar o } \\
\text { conhecimento de jovens a respeito de IST's e } \\
\text { sua prevenção. }\end{array}$ & $\begin{array}{l}344 \text { visitantes únicos do norte da Noruega desde } 10 \text { de } \\
\text { outubro de } 2012 ; 70,1 \% \text { deles são visitantes recorrentes e } \\
93,3 \% \text { acessaram o aplicativo da web por meio de } \\
\text { computadores. A média de páginas/visitas foi de } 23,9 \text { e } \\
\text { tempo total gasto no local de } 7 \text { minutos. Dados de saúde } \\
\text { pública relacionados com DSTs no Norte da Noruega } \\
\text { estão sendo coletados. }\end{array}$ & $\begin{array}{l}\text { Conclusão: Parece ser uma forma promissora de } \\
\text { encorajar os usuários a aprender mais sobre saúde } \\
\text { sexual de acordo com as taxas de retorno de } \\
\text { visitantes preliminares, a média de páginas visitadas } \\
\text { e o tempo gasto nelas. }\end{array}$ \\
\hline 38 & $\begin{array}{l}\text { MACÍAS E, et al. } \\
(2012)\end{array}$ & Estudo coorte & $\begin{array}{l}\text { O projeto aborda controle e proteção contra o } \\
\text { tabaco, exposição segura ao sol, exercício } \\
\text { físico e alimentação adequada. }\end{array}$ & $\begin{array}{l}\text { Avaliou a eficiência nas diferentes formas de interação } \\
\text { com o jogo e suas respectivas devolutivas pelos } \\
\text { participantes sendo o mouse o mais fácil e o mais familiar. }\end{array}$ & $\begin{array}{l}\text { Ajuda as crianças a aprender qual diferença entre } \\
\text { uma boa alimentação. Precisa ainda ser avaliado } \\
\text { melhor impacto sobre a atividade física promovida } \\
\text { através do projeto. }\end{array}$ \\
\hline
\end{tabular}




\section{DISCUSSÃO}

Os jogos digitais de saúde aumentam o envolvimento e tornam o ambiente de aprendizagem mais interativo, também oferecem habilidades práticas através de atividades de aprendizagem que são traduzíveis para a realidade. Sua natureza repetitiva é ideal para aprender. Fornecem feedback imediato, trazendo grandes benefícios (HARUNA H, et al., 2018).

Uma limitação trazida por Quintas-Hijós A, et al. (2020), foi o fato de nem toda a população ter acesso aos dispositivos eletrônicos, sejam eles computadores ou celulares com acesso à internet, e interroga se a solução para esse problema seriam campanhas para doação desses materiais pela população, ou se o mundo ainda não está pronto para a educação online, seja ela por jogos ou não.

Para ampliar o direito de acesso à internet, o Governo do Estado de São Paulo criou em julho de 2000 o AcessaSP, que é um programa de inclusão digital, com intuito de oferecer acesso gratuito à internet. Em dezembro de 2016 o programa foi reformulado pelo Decreto № 62.306/2016, para oferecer também conteúdos digitais que contribuíssem para o desenvolvimento pessoal, profissional e comunitário por meio da inclusão digital (ASSEMBLEIA LEGISLATIVA DO ESTADO DE SÃO PAULO, 2016).

Segundo Pourabbasi A, et al. (2020), a utilização de jogos sérios para ensino, traz mais benefícios para o aprendizado do que os métodos tradicionais, melhora as habilidades sociais, retenção de aprendizado e criatividade nos usuários. $O$ design de jogos sérios oferece domínios de grande alcance em aplicativos de saúde e está aumentando sua popularidade, especialmente com crianças e adolescentes, devido ao seu potencial para envolver e motivar os jogadores de maneira diferente de outras intervenções (ALMONDES KM e LEONARDO MEM ,2018).

Dessa forma, abre-se a possibilidade de efetuar trabalhos multidisciplinares, envolvendo pedagogos, psicopedagogos, médicos, enfermeiros, fisioterapeutas e outros profissionais para que o jogo coabite tanto na educação, sendo esta lúdica ou não, quanto na informação acadêmica. O jogo é para ser tanto educacional quanto desafiante e divertido (COIL DA, et al.,2017).

Jogos sérios e Gamificação proporcionam experiências poderosas e verdadeiras, simulando a realidade, ao fornecer ao usuário objetivos, desafios, resolução de problemas e regras, além de um valor interno claro e uma experiência interativa. Assim, podemos tornar a abordagem educativa mais interessante para o usuário, de forma que este incorpore com maior efetividade comportamentos de prevenção e promoção de saúde (MACÍAS E, et al., 2012).

Várias ferramentas foram desenvolvidas, como: sistemas de treinamento digital móvel conectados a sensores portáteis, jogos sérios e portais de pacientes para registros pessoais de saúde, que visam apoiar pacientes com condições crônicas e seus cuidadores na realização do ideal de autogerenciamento compartilhando o cuidado, entre a equipe de saúde, paciente e sua família, mas, centrando-o no paciente, corresponsabilizando-o por sua saúde (KLAASSEN R, et al.,2018).

Resultados preliminares mostram que o aplicativo da web de estilo de jogo, pode ser útil para encorajar os usuários a aprenderem mais sobre saúde sexual e Infecções Sexualmente Transmissíveis (ISTs) e, assim, mudar seus comportamentos de risco e prevenir infecções sexualmente transmissíveis (GABARROM $\mathrm{E}$, et al.,2013). Os jogos educativos oferecem anonimato e podem proporcionar oportunidade de esclarecer dúvidas, sem constrangimento, e, de conhecer, práticas específicas de sexo seguro em um ambiente virtual não socialmente ameaçador (BRÜLL P, et al.,2016).

A utilização dos jogos sérios tem como vantagem o impacto em diversos períodos de tempo, podendo ser imediato, como no caso de controle de dor no pós-operatório. O conhecimento de medicação para dor e estratégias de controle da dor melhoraram após o jogo (INGADOTTIR B, et al.,2017). Traz consigo a possibilidade de ser aplicado em diversos contextos como o intra-hospitalar. Segundo Carrión A, et al. (2019), obteve-se melhora de comunicação e coordenação motora em pacientes pediátricos que estavam internados e participaram do teste. Pode também ser usado em escolas como recurso pedagógico. Segundo Otero-Agra M, et al. (2019), observou-se melhor técnica de ressuscitação cardiopulmonar no grupo de treinamento com jogo em comparação ao grupo somente com aulas expositivas. 
Jogos sérios e gamificação podem também ser utilizados para efeitos a médio prazo, como no caso de adolescentes entrando na fase de atividade sexual. As duas abordagens de ensino inovadoras podem ser usadas para melhorar a educação em saúde sexual de estudantes adolescentes, pontuando sobre sexo seguro, com liguagem, exemplos e ambiente adequado para a população dessa faixa etária. Os métodos podem potencialmente contribuir na melhoria do comportamento de saúde sexual e no conhecimento dos adolescentes em problemas complexos de saúde sexual, incluindo vírus da imunodeficiência humana (HIV) / Síndrome de imunodeficiência adquirida (AIDS) e outras ISTs (HARUNA H, et al., 2019).

Pode também ser usado em pacientes com comorbidades como diabetes. Segundo Talley $\mathrm{MH}$, et al. (2019), o uso do jogo ao informar o paciente sobre sua doença, levou a transformação de uma consulta clínica comum para uma consulta inversa, que assim como em uma aula inversa o aluno passa a ser o foco do ensino, o paciente se tornou o foco de um aprendizado para melhor controle de sua doença crônica, mais um momento mostrando como o cuidado compartilhado pode trazer mais benefícios para pacientes com comorbidades, além de fazer com que as visitas ao médico sejam momentos de esclarecimento de dúvidas mais prazerosos.

A gamificação tem como vantagem o uso tanto para acometimentos biológicos como os supracitados, como para quadros psicossociais, em que se pode promover aprendizado social e emocional, com o propósito da melhora da percepção de bem-estar e saúde mental. Segundo Riordan BC e Scarf D (2016), indivíduos com Transtorno do Espectro Autista (TEA) sofrem com interações sociais cara-a-cara, mas eles ainda expressam desejo de se conectar socialmente. Jogar Minecraft pode ajudar esses jogadores a atingirem seus desejos sociais e obter seus efeitos positivos.

Segundo Carrión A, et al. (2019), percebeu-se melhora psicossocial, mesmo sem a interação com o professor, com melhora da feição e manteve os pacientes entretidos, os fazendo esquecer momentaneamente dos seus sintomas. Segundo Cejudo J, et al. (2020), pode-se ver melhora na percepção de qualidade de vida (relacionado à saúde), ao componente cognitivo da sensação de bem-estar, ao componente afetivo da sensação de bem-estar e à melhora da saúde mental.

O uso de jogos é vantajoso principalmente em atividades que impactam em pilares da saúde que terão repercussões em diversas áreas do cuidado, tanto a curto, médio e longo prazo. Segundo Azevedo J, et al. (2018), a gamificação permitiu a difusão de comportamento nutricional sustentável através da interação entre as famílias dos participantes na plataforma. Segundo Jones BA, et al. (2014), após a aplicação de um jogo sério com metas de alimentos a serem consumidos na cafeteria de uma escola que ia do jardim de infância até o fundamental II, durante um período de 13 dias letivos, o consumo de frutas aumentou em $66 \%$ e o consumo de vegetais em $44 \%$ acima dos níveis basais.

Também, segundo Holzmann SL, et al. (2019) e Villasana MV, et al. (2020), percebeu-se melhora do conhecimento sobre nutrição e $66,67 \%$ dos adolescentes avaliaram positivamente o uso de jogos em aparelhos móveis como recurso para melhora da alimentação e da prática de atividade física. Isso mostra que o recurso utilizado, mesmo em um período curto de tempo, obteve resultados positivos. Utilizando desta amostragem pode-se orientar novos estudos para a sedimentação desse novo hábito e da melhora de outros que se tinham parcialmente.

Segundo Blok AC, et al. (2019), adultos que vivem abaixo da linha de pobreza têm menos sucesso em tentativas de cessar tabagismo em comparação com adultos com condições socioeconômicas maiores. Com o uso de aplicativo para aparelho móvel pode-se alcançar e motivar populações mais vulneráveis a ter mais sucesso em mudanças comportamentais como em tentativas de cessar o tabagismo. Neste estudo $84,7 \%$ dos participantes responderam continuamente aos quizzes enviados por sms e $83 \%$ relataram que as mensagens motivacionais foram de grande ajuda, mas ainda, quando se trata de populações mais carentes ao acesso à informação e à tecnologia, os vieses dinheiro e acesso limitam os resultados, principalmente em populações maiores.

Evidências valiosas para uma nova abordagem na promoção de hábitos de sono saudáveis, com aplicações em contextos clínicos, educacionais e familiares, que poderiam diminuir futuros problemas de saúde e risco em doenças psiquiátricas, diminuindo a carga social de tratamentos para essas condições em crianças (ALMONDES KM e LEONARDO MEM, 2018). 
Na prevenção, podemos ter efeitos a curto, médio e longo prazo, assim, tanto na saúde do usuário, quanto no sistema de saúde, ampliando a otimização de seus serviços e diminuindo gastos por exacerbações recorrentes. A gamificação, sozinha ou com apoio social, aumentou a atividade física, a capacitação e diminuiu a utilização de serviços de saúde. Ao evoluir da tecnologia para jogos se tornou mais acessível o uso de aparelhos para rastreio de movimento, o que gerou um novo termo técnico, "exergame", uma mistura de exercícios e jogos (QUINTAS-HIJÓS A, et al.,2020).

Para melhorar a adesão aos cuidados, a atividade física e melhora dos parâmetros laboratoriais, depende da habilidade dos profissionais de saúde de incorporarem as preferências e necessidades dos pacientes no conteúdo do programa, ou seja, é para um bom resultado depende de uma boa relação médico-paciente para o sucesso. O conhecimento dos recursos, é importante para os profissionais de saúde, para que possam indicá-los adequadamente para cada faixa etária, otimizando a adesão e os resultados (STENOV V, et al., 2021).

Em contrapartida, Martos-Cabrera MB, et al (2020) diz que a tecnologia pode ser uma barreira em algumas populações, tanto os mais idosos, quanto às populações de baixa renda, e, especialmente os mais idosos e de baixo poder aquisitivo.

Quando se trata de prevenção de saúde, estima-se que grande parte da população tenha conhecimento dos recursos que o SUS (Sistema Único de Saúde) disponibiliza, mas a realidade não é essa, Darville G, et al. (2021) diz que a implantação de jogos traz efeitos positivos e significativos para o conhecimento, que tem implicações para a mudança do comportamento e que quando se trata de saúde pública os jogos tem se mostrado eficazes nas intervenções comportamentais, especialmente em imunizações, ISTs e prevenção de câncer. Impulsionando uma das características mais esquecidas do SUS, a promoção e prevenção em saúde. Porém, deve-se sempre levar em consideração a auto eficácia dos jogadores, pois as orientações são feitas, por escrito, nos próprios jogos e aplicativos, limitando o esclarecimento de dúvidas no momento em que elas surgem.

Os jogos, quando voltados para jovens, têm que ser planejados e desenvolvidos para esta faixa etária, assim como os jogos desenvolvidos para idosos, com layout específico, avatares e linguagens específicas, além de focar nos sinais e sintomas mais característicos de cada doença em cada grupo, caso contrário a eficiência e a adesão deixam a desejar. Gaspar JDS, et al. (2020) e Darville G, et al. (2021) dizem que quando há problemas de compreensão de leitura pelos mais jovens, estes deixam de aproveitar o momento de aprendizado como seria o esperado diminuindo a eficácia deste método (HUYNH E, et al., 2021). Já Fijacko N, et al. (2020) concorda com Gaspar JDS, et al. (2020) e Darville G, et al (2021), quando os aplicativos são desenvolvidos pensados nos mais jovens a adesão é grande e os resultados são positivos.

Após o início da pandemia do COVID-19 em março de 2020, a educação no mundo mudou, assim como seu acesso à saúde, se tratando de doenças crônicas ou mesmo endêmicas ou promoção e prevenção à saúde. Foi um momento que a internet e o ensino a distância tomaram grande evidência e importância. Dando espaço para jogos sérios e com isso aumentando as pesquisas acerca do assunto. Em um estudo com alunos, notou-se experiências significativas no aprendizado sobre ética, isso nos mostra que quando 0 jogo é desenvolvido para os mais jovens ele tem boa adesão, não importa em qual área seja. (ZAKARIA NS, et. al., 2020)

Segundo Holzmann SL, et al. (2019), existe a fragilidade na intervenção da amostra pequena, assim selecionando populações homogêneas com maioria masculina ou maioria caucasiana, causando vieses étnicos e sociodemográficos. Também, houve a não imparcialidade dos avaliadores que participaram tanto do grupo que sofreu a intervenção do jogo e o grupo controle, possibilitando vícios de ensino que diminuiu a distância entre o aprendizado dos dois grupos, estes vieses diminuem a acurácia da positividade dos resultados.

Sua maioria foram de estudos transversais que trouxeram resultados significativos para a intervenção imediata, mas não tiveram a amplitude do impacto de tal intervenção nas populações. Como em Blok AC, et al. (2019), obteve-se resultado positivo com a motivação para levar a população do estado de contemplação 
para o de ação, mas não conseguiu dar continuidade após as duas semanas de teste sobre o sucesso da manutenção da abstinência, impossibilitando o conhecimento dos resultados a longo prazo, e, da real efetividade do aprendizado nas mudanças de vida.

Alguns artigos utilizaram uma amostra pequena e/ou uma análise em um curto período de tempo devido a diminuição do acesso à página/app/plataforma. Para contornar essa dificuldade, Blok AC, et al (2019) utilizou lembretes para resgatar o usuário. Segundo Ryan J, et al. (2017), o uso de lembretes periódicos personalizados por e-mail pode aumentar o engajamento dos usuários, atraindo-os de volta à intervenção e ajudando a sustentar os comportamentos desejados.

Como é um recurso eletrônico, os jogos via browser, principalmente, enfrentam obstáculos comuns a todos os outros sites como conectividade, erros de programação entre outros. Algumas suposições feitas sobre como os usuários se conectam à plataforma não estavam satisfatórias na realidade, resultando em experiências de usuário inferiores às ótimas (KLAASSEN R, et al., 2018).

Outra limitação é desenvolver jogos direcionados para faixas etárias específicas, com os atrativos e linguagens adequados, e munir os profissionais de saúde para utilizá-los e trazer mais resultados do que os métodos tradicionais de educação (GASPAR JDS, et al., 2020 e POURABBASI A, et al., 2020).

\section{CONSIDERAÇÕES FINAIS}

A partir desta revisão pode-se concluir que mais estudos são necessários, abrangendo mais grupos, para tirar viés do ambiente, do acesso à informação e à dispositivos eletrônicos. Com a pandemia do COVID-19 os jogos passaram a ser mais utilizados para o ensino, mas ainda é pouco difundida, principalmente para o ensino em saúde. Até o momento os resultados foram positivos, permitindo prosseguir estudando maneiras de melhor difusão da gamificação no aprendizado. Para tal, é preciso que o docente reconheça o potencial desta nova ferramenta/instrumento, permitindo ampliação do vínculo com os discentes, provendo acesso amplo às novas tecnologias de ensino, além de reforçar as políticas públicas de acessibilidade às tecnologias, principalmente às populações mais carentes e prejudicadas, permitindo igualdade de acesso e ensino a todas as comunidades. Apesar destas condições, os jogos vêm apresentando resultados positivos.

\section{REFERÊNCIAS}

1. ALLAN A. The effect of social support features and gamification on a Web-based intervention for rheumatoid arthritis patients: randomized controlled trial. Journal of Medical Internet Research, 2015; 17.

2. ALMONDES KM, LEONARDO MEM. Study Protocol of Sleep Education Tool for Children: Serious Game "Perfect Bedroom: Learn to Sleep Well". Frontiers of Psycholgy, 2018; 9(1016).

3. ASSEMBLEIA LEGISLATIVA DO ESTADO DE SÃO PAULO. Decreto № 62.306. 2016. Disponível em: https://www.al.sp.gov.br/repositorio/legislacao/decreto/2016/decreto-62306-14.12.2016.html. Acessado em: 25 de maio de 2021.

4. AZEVEDO J, et al. A Web-Based Gamification Program to Improve Nutrition Literacy in Families of 3- to 5-Year-Old Children: The Nutriscience Project. Journal of Nutrition Education and Behavior, 2018; 1-9.

5. BELOGIANNI K, BALDWIN C. Types of Interventions Targeting Dietary, Physical Activity, and Weight-Related Outcomes among University Students: A Systematic Review of Systematic Reviews. 2019;10(5):848-863

6. BLOK AC, et al. Gamification to Motivate the Unmotivated Smoker: The "Take a Break" Digital Health Intervention. Games for Health Journal, 2019; 275-284.

7. BURGESS JD, et al. Cool Runnings - an app-based intervention for reducing hot drink scalds: study protocol for a randomised controlled trial. Trials, 2016; 17:388.

8. BRÜLL P, et al. Gaming for Safer Sex: Young German and Turkish People Report No Specific Culture-Related Preferences Toward Educational Games Promoting Safer Sex. Games Health Journal, 2016; 5(6).

9. CARRIÓN A, et al. Tangibot: a collaborative multiplayer game for pediatric patients. International Journal of Medical Informatics, 2019; 1-40.

10. CEJUDO J, et al. Promoting Social and Emotional Learning and Subjective Well-Being: Impact of the "Aislados" Intervention Program in Adolescents. Int. Journal of Environment Research and Public Health, 2020; 17:609.

11. COIL DA, et al. Gut Check: The evolution of an educational board game. PLoS Biol, 2017; 15(4). 
12. DARVILLE G, et al. Utilizing Theories and Evaluation in Digital Gaming Interventions to Increase Human Papillomavirus Vaccination Among Young Males: Qualitative Study. JMIR Serious Games, 2021; 9(1).

13. DESMET, A et al. A Systematic Review and Meta-alaysis of Interventions for Sexual Health Promotion Involving Serious Digital Games. Games for Health Journal 2015; 4 (2)

14. FIJACKO N, et al. The Effects of Gamification and Oral Self-Care on Oral Hygiene in Children: Systematic Search in App Stores and Evaluation of Apps. JMIR Mhealth Uhealth, 2020; 8(7).

15. FLEMING TM, et al. Maximizing the Impacto of e-therapy and Serious Gaming: Time for a Paradigm Shift.Front Psychiatry. 2016; 7:65.

16. GABARRON E, et al. Gamification strategy on prevention of STDs for youth. Stud in Health Technology and Informatics. 2013; 192:1066.

17. GASPAR JDS, et al. A Mobile Serious Game About the Pandemic (COVID-19 - Did You Know?): Design and Evaluation Study. JMIR Serious Games, 2020; 8(4).

18. HARUNA H, et al. Improving Sexual Health Education Programs for Adolescent Students through Game-Based Learning and Gamification. International Journal of Environment Research and Public Health, 2018; 15(9).

19. HARUNA H, et al. Initial Validation of the MAKE Framework: A Comprehensive Instrument for Evaluating the Efficacy of Game-Based Learning and Gamification in Adolescent Sexual Health Literacy. Annals of Global Health. 2019; 85(1).

20. HIGHTOW-WEIDMAN, LB, et al. The future of digital games for HIV prevention and care. Current Opinion in HIV and AIDS, 2017; 12(5), 501-507.

21. HOLZMANN SL, et al. Short-Term Effects of the Serious Game "Fit, Food, Fun" on Nutritional Knowledge: A Pilot Study among Children and Adolescents. Nutrients, 2019; 11, 2031.

22. HUYNH E, et al. Designing Narrative-Focused Role-Playing Games for Visualization Literacy in Young Children. IEEE Transactions on Visualization and Computer Graphics, 2021; 27(2): 924-934.

23. INGADOTTIR B, et al. Development, Usability, and Efficacy of a Serious Game to Help Patients Learn About Pain Management After Surgery: An Evaluation Study. JMIR Serious Games, 2017; 5(2).

24. JONES BA, et al. Gamification of dietary decision-making in an elementary-school cafeteria. PLoS One, 2014; 9(4).

25. KLAASSEN R, et al. Design and Evaluation of a Pervasive Coaching and Gamification Platform for Young Diabetes Patients. Sensors (Basel), 2018; 18(2).

26. MACÍAS E, et al. Glooveth: healthy living, fun and serious gaming. Stud in Health Technology and Informatics, 2012; 172: $180-184$.

27. MARTOS-CABRERA MB, et al. Games and Health Education for Diabetes Control: A Systematic Review with MetaAnalysis. Healthcare, 2020; 8(4):399.

28. MONTANER J, et al. Tangibot: a collaborative multiplayer game for pediatric patients. International Journal of Medical Informatics, 2019; 132.

29. OTERO-AGRA M, et al. Let the kids play: gamification as a CPR training methodology in secondary school students. A quasi-experimental manikin simulation study. Emergency Medicine Journal, 2019; 653-659.

30. POURABBASI A, et al. Playing With Little Behaviors; Physical Activity Promotion by Gamified Education in Young Boys. International Journal of Preventive Medicine, 2020; 11:71.

31. QUINTAS-HIJÓS A, et al. Analysis of the applicability and utility of a gamified didactics with exergames at primary schools: Qualitative findings from a natural experiment. PLoS ONE, 2020; 15(4).

32. RIORDAN BC, SCARF D. Crafting minds and communities with Minecraft. F1000Research, 2016; 5:2339.

33. RYAN J, et al. Engagement, Compliance and Retention With a Gamified Online Social Networking Physical Activity Intervention. Translational Behavior Medicine, 2017; 7(4).

34. RYU JH, et al. The Effect of Gamification through a Virtual Reality on Preoperative Anxiety in Pediatric Patients Undergoing General Anesthesia: A Prospective, Randomized, and Controlled Trial. Journal of Clinical Medicine, 2018; 7(9), 284.

35. STAPINSKI LA, et al. Development and Evaluation of 'Pure Rush': An Online Serious Game for Drug Eucation. Drug and alcohol review, 2018; 37: 420-428.

36. STENOV $V$ et al. Testing an Analogue Game to Promote Peer Support and Person-centredness in Education for People with Diabetes: A Realist Evalution. Nursing Open, 2021; 8:2536-2550.

37. TALLEY MHI, et al. Kaizen: Interactive Gaming for Diabetes Patient Education. Games for Health Journal, 2019 ; $423-$ 431.

38. VILLASANA MV, et al. Promotion of Healthy Nutrition and Physical Activity Lifestyles for Teenagers: A Systematic Literature Review of The Current Methodologies. Journal of Personalized Medicine, 2020; 10-12.

39. THENG YL, et al. The Use of Videogame, Gamification, and Virtual Environments in the Self-Management of Diabetes: A Systematic Review of Evidence. Games for Health Journal.Oct, 2015; 352-361.

40. ZAKARIA NS, et al. Assessing Ethoshunt as a Gamification-Based Mobile App in Ethics Education: Pilot MixedMethods Study. JMIR Serious Games, 2020; 8(3). 